\title{
Control of Traffic Intensity in Hyperexponential and Mixed Erlang Queueing Systems with a Method Based on SPRT
}

\author{
Müjgan Zobu' ${ }^{1}$ and Vedat Sağlam ${ }^{2}$ \\ ${ }^{1}$ Department of Statistics, Amasya University, 05000 Amasya, Turkey \\ ${ }^{2}$ Department of Statistics, Ondokuz Mayıs University, 55139 Samsun, Turkey \\ Correspondence should be addressed to Müjgan Zobu; mujganzobu@hotmail.com
}

Received 6 December 2012; Revised 30 May 2013; Accepted 10 June 2013

Academic Editor: Wuquan Li

Copyright (C) 2013 M. Zobu and V. Sağlam. This is an open access article distributed under the Creative Commons Attribution License, which permits unrestricted use, distribution, and reproduction in any medium, provided the original work is properly cited.

\begin{abstract}
The control of traffic intensity $(\rho)$ is one of the important problems in the study of queueing systems. Rao et al. (1984) developed a method to detect changes in the traffic intensity in queueing systems of the $M / G / 1$ and $G I / M / c$ types based on the Sequential Probability Ratio Test (SPRT). In this paper, SPRT is theoretically investigated for two different phase-type queueing systems which consist of hyperexponential and mixed Erlang. Also, for testing $H_{0}: \rho=\rho_{0}$ against $H_{1}: \rho=\rho_{1}$, Operating Characteristic (OC) and Average Sample Number (ASN) functions are obtained with numerical methods using multipoint derivative equations according to different situations of $\alpha$ and $\beta$ type errors. Afterward, numerical illustrations for each model are provided with Matlab programming.
\end{abstract}

\section{Introduction}

There are very few studies on the sequential detection of parameter changes of queueing systems in the literature. The theory of SPRT for a sequence of observations forming a finite Markov chain was given in [1]. After, based upon the theory of [1] was discussed statistical quality control and SPRT procedures for the control of traffic intensity in [26]. This method aims to detect changes in traffic intensity by observing only the number of customers in the system at successive departure epochs $\mathbb{Q}_{n}$, which are embedded Markov points. Recently, a SPRT to regulate the traffic intensity based on the number of arrivals during the $n$th service periods for $M / E_{k} / 1$ queue was proposed in [7] and an autoregressive process based on the number of customers at the departure point and its application to the queueing model were given in [8]. In this paper, the method based on the SPRT is first examined theoretically for the two different queueing phase types. Afterward, the observations obtained for different samples model are evaluated for each of the models by simulation through MATLAB 7.10.0 (R2010a) programming.

\section{Queueing Process and SPRT}

In many queueing applications, a performance characteristic of great importance and interest is the traffic intensity $\rho$ (the ratio of mean arrival rate $\lambda$ to mean service rate $\mu$ ). The purpose of this test is to determine as quickly as possible the changes in traffic intensity and to take the appropriate corrective actions. With this objective, a procedure has been developed for testing the hypothesis $H_{0}: \rho=\rho_{0}$ against $H_{1}: \rho=\rho_{1}$ using Wald's SPRT for the systems $M / G / 1$ and $G I / M / c$, in which queue length processes have imbedded Markov chains $\left\{Q_{n}\right\}$ in [6]. This procedure is explained as follows.

Consider the single server queue where arrivals occur according to a Poisson process with rate $\lambda$ per unit time. The service times of customers are independent identically distributed (i.i.d.) random variables with the distribution $B(x)$. For this system, the queue lengths at service completion points form an imbedded Markov chain. $Q_{n}$ will be the number of customers left behind by the $n$th departing customer. The capacity of the queueing system is restricted 
to $N$. Then the transition probability matrix of the imbedded Markov chain is given by

$$
\begin{aligned}
& P=\left\{P_{i j}(\rho)\right\} \\
& \begin{array}{c}
0 \\
= \\
\quad \\
\cdot \\
\vdots \\
\cdot \\
\cdot
\end{array} \quad\left[\begin{array}{ccccc}
0 & 1 & 2 & \cdots & N-1 \\
k_{0} & k_{1} & k_{2} & \ldots & 1-\sum_{0}^{k-2} k_{j} \\
k_{0} & k_{1} & k_{2} & \ldots & 1-\sum_{0}^{k-2} k_{j} \\
0 & k_{0} & k_{1} & \ldots & 1-\sum_{0}^{k-3} k_{j} \\
0 & 0 & k_{0} & \ldots & \cdot \\
\vdots & \vdots & \vdots & & \cdot \\
0 & 0 & 0 & \ldots & \cdot \\
0 & 0 & 0 & \ldots & \cdot \\
0 & 0 & 0 & \ldots & 1-k_{0}
\end{array}\right]
\end{aligned}
$$

where $k_{n}=P\{n$ arrivals during a service period $\}=$ $\int_{0}^{\infty} e^{-\lambda t}\left((\lambda t)^{j} / j !\right) d B(t)$.

Let $t_{0}<t_{1}<t_{2}<\cdots<t_{n}$ be the set of time points at which the $Q(t)$ process exhibits the Markov property; namely,

$$
\begin{gathered}
P\left\{\mathbb{Q}\left(t_{n+1}\right)=j \backslash \mathbb{Q}\left(t_{n}\right)=i, \mathbb{Q}\left(t_{n-1}\right)=i_{n-1}, \ldots, \mathbb{Q}\left(t_{0}\right)=i_{0}\right\} \\
=P\left\{Q\left(t_{n+1}\right)=j \backslash \mathbb{Q}\left(t_{n}\right)=i\right\} .
\end{gathered}
$$

In the $M / G / 1$ queue, $t_{n}$ is the time of service completion of the $n$th customer and, in the case of the $G I / M / c$ queue, $t_{n}$ is the arrival epoch of the $n$th customer. In the case of the $M / M / c / N$ queue, though the process is in Markovian continuous time, for purposes of control it is easier to examine the process at arrival points.

For simplicity, we will use the notation $Q_{n}=Q_{2}\left(t_{n}\right)$. Denoted by $P=\left\{P_{i j}(\rho)\right\}$ the transition probability matrix of the Markov chain $\left\{Q_{n}\right\}$ defined over state space $E=$ $\{0,1,2, \ldots, N\}$, where

$$
P_{i j}(\rho)=P\left\{Q_{n+1}=j \backslash Q_{n}=i ; \rho\right\} .
$$

It is assumed that $P$ is known except for the value of the parameter $\rho$. The problem is to test the hypothesis $H_{0}: \rho=\rho_{0}$ against $H_{1}: \rho=\rho_{1}$.

Consider the sequence of observations $Q_{0}, Q_{1}, Q_{2}, \ldots, Q_{n}$. The joint probability of observing this sequence under $H_{0}$ and $H_{1}$ is given by

$$
\operatorname{Pr}\left\{\mathbb{Q}_{0}, \mathbb{Q}_{1}, \mathcal{Q}_{2}, \ldots, \mathbb{Q}_{a} ; \rho_{i}\right\}=P\left(\mathbb{Q}_{0} ; \rho_{i}\right) \prod_{j=1}^{n} P\left(\mathbb{Q}_{j} \backslash \mathbb{Q}_{j-1} ; \rho_{i}\right)
$$

Then the likelihood ratio is

$$
\begin{aligned}
& L=\frac{P\left(\widehat{Q}_{0} ; \rho_{1}\right) \prod_{j=1}^{n} P\left(\widehat{Q}_{j} \backslash Q_{j-1} ; \rho_{1}\right)}{P\left(Q_{0} ; \rho_{0}\right) \prod_{j=1}^{n} P\left(Q_{j} \backslash Q_{j-1} ; \rho_{0}\right)} . \\
& Z_{0}=\ln \frac{P\left(Q_{0} ; \rho_{1}\right)}{P\left(Q_{0} ; \rho_{0}\right)}, \\
& Z_{r}=\ln \frac{P\left(\mathscr{Q}_{r} \backslash \mathcal{Q}_{r-1} ; \rho_{1}\right)}{P\left(\mathcal{Q}_{r} \backslash \mathcal{Q}_{r-1} ; \rho_{0}\right)}, \quad(r \geq 1) \text {. }
\end{aligned}
$$

Let $A=(1-\beta) / \alpha$ and $B=\beta /(1-\alpha)$, where $\alpha$ and $\beta$ are the probabilities of the errors of the first and second types. Then, Wald's SPRT [9] to test $H_{0}: \rho=\rho_{0}$ against $H_{1}: \rho=\rho_{1}$ becomes as follows. 1 ,

Observe $\left\{Q_{i}\right\}(i=0,1,2, \ldots)$ successively and at stage $n \geq$

(1) accept $H_{0}$ if $\sum_{0}^{n} Z_{r} \leq \ln B$;

(2) accept $H_{1}$ if $\sum_{0}^{n} Z_{r} \geq \ln A$;

(3) continue by observing $Q_{n+1}$ if $\ln B<\sum_{0}^{n} Z_{r}<\ln A$.

If we assume $Q_{0}=i_{0}$ is specified and denote by $n_{i j}$ the number of transitions $i \rightarrow j$ up to and including the $n$th transition, then the likelihood ratio given in (5) reduces to

$$
\begin{gathered}
L=\frac{\prod_{i, j} P_{i j}^{n_{i j}}\left(\rho_{1}\right)}{\prod_{i, j} P_{i j}^{n_{i j}}\left(\rho_{0}\right)}, \\
\ln L=\sum_{i, j} n_{i j} \ln \frac{P_{i j}\left(\rho_{1}\right)}{P_{i j}\left(\rho_{0}\right)} .
\end{gathered}
$$

Then the SPRT for testing the hypothesis $H_{0}: \rho=\rho_{0}$ against $H_{1}: \rho=\rho_{1}$ will have its continuation region

$$
\ln B<\sum_{i, j} n_{i j} \ln \frac{P_{i j}\left(\rho_{1}\right)}{P_{i j}\left(\rho_{0}\right)}<\ln A .
$$

In the case of $M / E_{k} / 1, E_{k} / M / c$, and $M / M / c / N$ queues, we will show in the sequel that the logarithm of the likelihood ratio can be written in the form

$$
\ln L=a n+\sum_{i, j} n_{i j} c_{i j}
$$

where $a, c_{i j}$, and $P_{i j}$ are constants depending upon the parameters $\rho_{1}, \rho_{0}$, and the transition probabilities $P_{i j}$. Thus (9) reduces to

$$
\ln B-a n<\sum_{i, j} n_{i j} c_{i j}<\ln A-a n .
$$

From this it follows that the continuation region of the test is bounded by straight lines $\ln A-a n$ ve $\ln B-a n$.

\section{Operating Characteristic and Average Sample Number}

Approximate formulas for the OC and ASN functions are given in [1]. In order to evaluate the OC and ASN functions, 
$\lambda_{0}(t, \rho)$ is determined as the largest real positive latent root of the matrix

$$
P(t)=\left\{p_{i j}(\rho)\left[\frac{p_{i j}\left(\rho_{1}\right)}{p_{i j}\left(\rho_{0}\right)}\right]^{t}\right\},
$$

its derivate at $t=0$ and the nonzero root $t(\rho)$ of the equation $\lambda_{0}(t, \rho)=1$.

A numerical search technique was employed to find the root in [10]. For evaluating the derivate of $\lambda_{0}(t, \rho)$ at $t=$ 0 , the following five-point numerical differentiation formula has been used:

$$
\begin{gathered}
\lambda_{0}^{\prime}(t, \rho) \cong \frac{1}{12 h}\left\{\lambda_{-2}-8 \lambda_{-1}+8 \lambda_{1}-\lambda_{2}\right\}, \\
\lambda_{0}^{\prime \prime}(t, \rho) \cong \frac{1}{12 h^{2}}\left\{-\lambda_{-2}+16 \lambda_{-1}-30 \lambda_{0}+16 h_{1}-\lambda_{2}\right\} .
\end{gathered}
$$

When the stated space of $\left\{\mathscr{Q}_{n}\right\}$ is finite, the OC function for the SPRT can be obtained as

$$
\begin{aligned}
L(\rho) & \cong \frac{A^{t_{0}(\rho)}-1}{A^{t_{0}(\rho)}-B^{t_{0}(\rho)}} \quad \text { if } t_{0}(\rho) \neq 0 \\
& \cong \frac{\ln A}{\ln A-\ln B} \quad \text { if } t_{0}(\rho)=0,
\end{aligned}
$$

where $t_{0}(\rho)$ is the nonzero real root of the equation $\lambda_{0}(t, \rho)=$ 1. The ASN can then be obtained as

$$
\begin{aligned}
E(n ; \rho) & \cong \frac{L(\rho) \ln B+\{1-L(\rho)\} \ln A}{\lambda_{0}^{\prime}(0)} \text { if } \lambda_{0}^{\prime} \neq 0 \\
& \cong \frac{L(\rho)\{\ln B\}^{2}+\{1-L(\rho)\}\{\ln A\}^{2}}{\lambda_{0}^{\prime \prime}} \text { if } \lambda_{0}^{\prime}=0 .
\end{aligned}
$$

\section{SPRT for Phase-Type Distribution}

The exponential distribution is very widely used in performance modelling. The reason, of course, is that mathematical tractability flows from the memoryless property of this distribution. But sometimes mathematical tractability is not sufficient to overcome the need for a model process in which the exponential distribution is simply not adequate. This leads us to explore ways in which we can develop more general distributions while maintaining some of the tractability of the exponential. This is precisely what phase-type distributions permit us to do [11].

4.1. Hyperexponential-2 Service Model: The $M / H_{2} / 1$ Queue. Consider the configuration presented in Figure 1, in which $\alpha_{1}$ is the probability that the upper phase is taken and $\alpha_{2}=$ $1-\alpha_{1}$ the probability that the lower phase is taken. If such a distribution is used to model a service facility, then a customer entering a service will, with probability $\alpha_{1}$, receive the service that is exponentially distributed with parameter $\mu_{1}$ and then exit the server or else with probability $\alpha_{2}$ receive the service that is exponentially distributed with parameter $\mu_{2}$ and then exit the server. Once again, only one customer

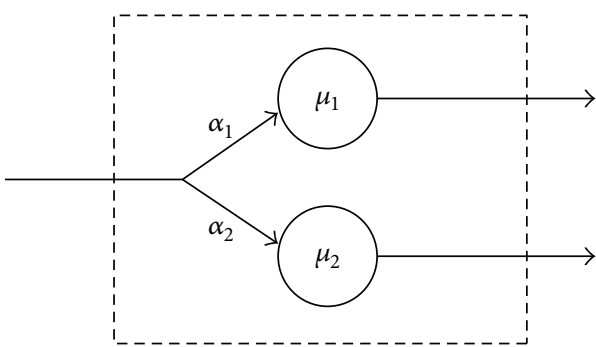

FIGURE 1: Two exponential phases in parallel.

can be in the process of receiving service at any one time; that is, both phases cannot be active at the same time [11].

Theorem 1. The density function of the service time is given by

$$
\frac{d B(x)}{d x}=\left(\alpha_{1} \mu_{1} e^{-\mu_{1} x}+\alpha_{2} \mu_{2} e^{-\mu_{2} x}\right), \quad(x \geq 0) .
$$

In this case, one has

$$
\begin{aligned}
k_{n}= & \alpha_{1}\left(\frac{\rho_{1}}{\rho_{1}+1}\right)^{n}\left(\frac{1}{\rho_{1}+1}\right) \\
& +\alpha_{2}\left(\frac{\rho_{2}}{\rho_{2}+1}\right)^{n}\left(\frac{1}{\rho_{2}+1}\right),
\end{aligned}
$$

where $k_{n}=P\{n$ arrivals during a service period $\}$.

Proof. Consider

$$
\begin{aligned}
k_{n}= & \int_{0}^{\infty} \frac{e^{-\lambda t}(\lambda t)^{n}}{n !}\left(\alpha_{1} \mu_{1} e^{-\mu_{1} t}+\alpha_{2} \mu_{2} e^{-\mu_{2} t}\right) d(t) \\
= & \int_{0}^{\infty} \frac{e^{-\lambda t}(\lambda t)^{n}}{n !} \alpha_{1} \mu_{1} e^{-\mu_{1} t} d(t) \\
& +\int_{0}^{\infty} \frac{e^{-\lambda t}(\lambda t)^{n}}{n !} \alpha_{2} \mu_{2} e^{-\mu_{2} t} d(t) \\
= & \frac{\alpha_{1} \mu_{1} \lambda^{n}}{n !} \int_{0}^{\infty} e^{-\lambda t} t^{n} e^{-\mu_{1} t} d(t) \\
& +\frac{\alpha_{2} \mu_{2} \lambda^{n}}{n !} \int_{0}^{\infty} e^{-\lambda t} t^{n} e^{-\mu_{2} t} d(t) \\
= & \frac{\alpha_{1} \mu_{1} \lambda^{n}}{n !} \int_{0}^{\infty} e^{-t\left(\lambda+\mu_{1}\right)} t^{n} d(t) \\
& +\frac{\alpha_{2} \mu_{2} \lambda^{n}}{n !} \int_{0}^{\infty} e^{-t\left(\lambda+\mu_{2}\right)} t^{n} d(t) \\
= & \frac{\alpha_{1} \mu_{1} \lambda^{n}}{n !}\left(\frac{1}{\lambda+\mu_{1}}\right)^{n+1} \Gamma(n+1) \\
& +\frac{\alpha_{2} \mu_{2} \lambda^{n}}{n !}\left(\frac{1}{\lambda+\mu_{2}}\right)^{n+1} \Gamma(n+1) \\
& \Gamma(t)
\end{aligned}
$$


4

Mathematical Problems in Engineering

$$
\begin{aligned}
= & \frac{\alpha_{1} \mu_{1} \lambda^{n} n !}{n !}\left(\frac{1}{\lambda+\mu_{1}}\right)^{n+1}+\frac{\alpha_{2} \mu_{2} \lambda^{n} n !}{n !}\left(\frac{1}{\lambda+\mu_{2}}\right)^{n+1} \\
= & \alpha_{1}\left(\frac{\lambda}{\lambda+\mu_{1}}\right)^{n}\left(\frac{\mu_{1}}{\lambda+\mu_{1}}\right)+\alpha_{2}\left(\frac{\lambda}{\lambda+\mu_{2}}\right)^{n}\left(\frac{\mu_{2}}{\lambda+\mu_{2}}\right) \\
= & \alpha_{1}\left(\frac{\lambda / \mu_{1}}{\lambda / \mu_{1}+\mu_{1} / \mu_{1}}\right)^{n}\left(\frac{\mu_{1} / \mu_{1}}{\lambda / \mu_{1}+\mu_{1} / \mu_{1}}\right) \\
& +\alpha_{2}\left(\frac{\lambda / \mu_{2}}{\lambda / \mu_{2}+\mu_{2} / \mu_{2}}\right)^{n}\left(\frac{\mu_{2} / \mu_{2}}{\lambda / \mu_{2}+\mu_{2} / \mu_{2}}\right) \\
= & \alpha_{1}\left(\frac{\rho_{1}}{\rho_{1}+1}\right)^{n}\left(\frac{1}{\rho_{1}+1}\right)+\alpha_{2}\left(\frac{\rho_{2}}{\rho_{2}+1}\right)^{n}\left(\frac{1}{\rho_{2}+1}\right) .
\end{aligned}
$$

Here

$$
\begin{gathered}
P_{i j}(\rho)=k_{j-i+1}, \quad i=1,2, \ldots, N, \\
j=0,1, \ldots, N-1, \quad j \geq i-1, \\
P_{0 j}(\rho)=k_{j}, \quad j=0,1, \ldots, N-1, \\
P_{0 N}(\rho)=1-\sum_{0}^{N-1} k_{n}, \\
P_{i N}(\rho)=1-\sum_{0}^{N-i} k_{n}, \quad i=1,2, \ldots, N, \\
P_{i N}(\rho)=1-\sum_{r=0}^{N-i} \alpha_{1}\left(\frac{\rho_{1}}{\rho_{1}+1}\right)^{r}\left(\frac{1}{\rho_{1}+1}\right) \\
+\alpha_{2}\left(\frac{\rho_{2}}{\rho_{2}+1}\right)^{r}\left(\frac{1}{\rho_{2}+1}\right) .
\end{gathered}
$$

With these values for $P_{i j}(\rho)$, the logarithm of the likelihood ratio will be

$$
\begin{aligned}
\ln L=\sum_{i, j} n_{i j} \ln \frac{P_{i j}\left(\rho_{1}\right)}{P_{i j}\left(\rho_{0}\right)} \\
\ln L=\sum_{i, j} n_{i j} \ln \left(\left(\alpha_{1}\left(\frac{\rho_{1(1)}}{\rho_{1(1)}+1}\right)^{n}\left(\frac{1}{\rho_{1(1)}+1}\right)\right.\right. \\
\left.+\alpha_{2}\left(\frac{\rho_{2(1)}}{\rho_{2(1)}+1}\right)^{n}\left(\frac{1}{\rho_{2(1)}+1}\right)\right) \\
\times\left(\alpha_{1}\left(\frac{\rho_{1(0)}}{\rho_{1(0)}+1}\right)^{n}\left(\frac{1}{\rho_{1(0)}+1}\right)\right. \\
\left.\left.+\alpha_{2}\left(\frac{\rho_{2(0)}}{\rho_{2(0)}+1}\right)^{n}\left(\frac{1}{\rho_{2(0)}+1}\right)\right)^{-1}\right)
\end{aligned}
$$

where

$$
\begin{gathered}
c_{i j}=\ln \left(\left(\alpha_{1}\left(\frac{\rho_{1(1)}}{\rho_{1(1)}+1}\right)^{j-i+1}\left(\frac{1}{\rho_{1(1)}+1}\right)\right.\right. \\
\left.\quad+\alpha_{2}\left(\frac{\rho_{2(1)}}{\rho_{2(1)}+1}\right)^{j-i+1}\left(\frac{1}{\rho_{2(1)}+1}\right)\right) \\
\times\left(\alpha_{1}\left(\frac{\rho_{1(0)}}{\rho_{1(0)}+1}\right)^{j-i+1}\left(\frac{1}{\rho_{1(0)}+1}\right)\right. \\
\left.\left.\quad+\alpha_{2}\left(\frac{\rho_{2(0)}}{\rho_{2(0)}+1}\right)^{j-i+1}\left(\frac{1}{\rho_{2(0)}+1}\right)\right)^{-1}\right), \\
i=1,2, \ldots, N, j=0,1, \ldots, N-1, j \geq i-1,
\end{gathered}
$$

$$
\begin{aligned}
c_{0 j}=\ln \left(\left(\alpha_{1}\left(\frac{\rho_{1(1)}}{\rho_{1(1)}+1}\right)^{j}\left(\frac{1}{\rho_{1(1)}+1}\right)\right.\right. \\
\left.\quad+\alpha_{2}\left(\frac{\rho_{2(1)}}{\rho_{2(1)}+1}\right)^{j}\left(\frac{1}{\rho_{2(1)}+1}\right)\right) \\
\quad \times\left(\alpha_{1}\left(\frac{\rho_{1(0)}}{\rho_{1(0)}+1}\right)^{j}\left(\frac{1}{\rho_{1(0)}+1}\right)\right. \\
\left.\left.\quad+\alpha_{2}\left(\frac{\rho_{2(0)}}{\rho_{2(0)}+1}\right)^{j}\left(\frac{1}{\rho_{2(0)}+1}\right)\right)^{-1}\right), \\
j=0,1, \ldots, N-1,
\end{aligned}
$$

$c_{i N}$

$$
\begin{gathered}
=\ln \left\{\left(1-\sum_{r=0}^{N-i}\left(\alpha_{1}\left(\frac{\rho_{1(1)}}{\rho_{1(1)}+1}\right)^{r}\left(\frac{1}{\rho_{1(1)}+1}\right)\right.\right.\right. \\
\left.\left.+\alpha_{2}\left(\frac{\rho_{2(1)}}{\rho_{2(1)}+1}\right)^{r}\left(\frac{1}{\rho_{2(1)}+1}\right)\right)\right) \\
\times\left(1-\sum_{r=0}^{N-i}\left(\alpha_{1}\left(\frac{\rho_{0(1)}}{\rho_{0(1)}+1}\right)^{r}\left(\frac{1}{\rho_{0(1)}+1}\right)\right.\right. \\
\left.\left.\left.+\alpha_{2}\left(\frac{\rho_{0(1)}}{\rho_{0(1)}+1}\right)^{r}\left(\frac{1}{\rho_{0(1)}+1}\right)\right)\right)^{-1}\right\}, \\
c_{0 N}=c_{1 N} .
\end{gathered}
$$

4.2. Mixed Erlang-k Service Model: The $M / \operatorname{mix}_{k} / 1$ Queue. This is illustrated in Figure 2, where, with probability $\alpha$, the top series of $k-1$ exponential phases is taken and, with probability $1-\alpha$, the bottom series of $k$ phases is taken. 


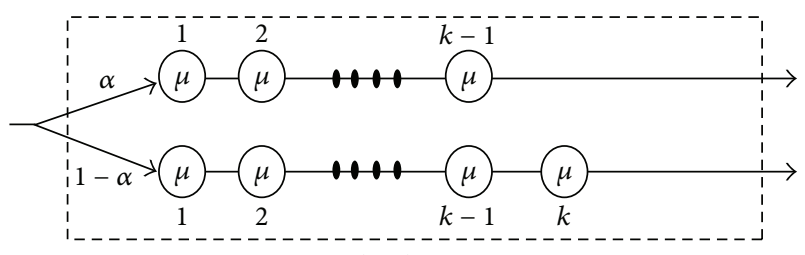

FIGURE 2: Mixed Erlang representation.

Theorem 2. The density function of the service time is given by

$$
\begin{aligned}
\frac{d B(x)}{d x}= & \alpha \frac{\mu(\mu x)^{k-2} e^{-\mu x}}{(k-2) !} \\
& +(1-\alpha) \frac{\mu(\mu x)^{k-1} e^{-\mu x}}{(k-1) !}, \quad(x \geq 0),
\end{aligned}
$$

where $k \geq 2$ is an integer.

In this case, one has

$$
\begin{aligned}
k_{n}= & \left(\frac{\rho}{\rho+k}\right)^{n}\left(\frac{k}{\rho+k}\right)^{k-1} \frac{(n+k-2) !}{n !(k-2) !} \\
& \times\left(\alpha+(1-\alpha) \frac{(n+k-1)}{(k-1)}\left(\frac{k}{\rho+k}\right)\right),
\end{aligned}
$$

where $k_{n}=P\{n$ arrivals during a service period $\}=$ $\int_{0}^{\infty} e^{-\lambda t}\left((\lambda t)^{j} / j !\right) d B(t)$.

Proof. Consider

$$
\begin{aligned}
& k_{n}=\int_{0}^{\infty} \frac{e^{-\lambda t}(\lambda t)^{n}}{n !} \\
& \times\left(\alpha \frac{(k \mu t)^{k-2}}{(k-2) !} e^{-k t x} k \mu+(1-\alpha) \frac{(k \mu t)^{k-1}}{(k-1) !} e^{-k \mu t} k \mu\right) d(t) \\
& =\int_{0}^{\infty} \frac{e^{-\lambda t}(\lambda t)^{n}}{n !} \alpha \frac{(k \mu t)^{k-2}}{(k-2) !} e^{-k t x} k \mu d(t) \\
& +\int_{0}^{\infty} \frac{e^{-\lambda t}(\lambda t)^{n}}{n !}(1-\alpha) \frac{(k \mu t)^{k-1}}{(k-1) !} e^{-k t x} k \mu d(t) \\
& =\frac{\alpha k \mu \lambda^{n}(k \mu)^{k-2}}{n !(k-2) !} \int_{0}^{\infty} e^{-\lambda t} t^{n} e^{-k \mu t} t^{k-2} d(t) \\
& +\frac{(1-\alpha) k \mu \lambda^{n}(k \mu)^{k-1}}{n !(k-1) !} \int_{0}^{\infty} e^{-\lambda t} t^{n} e^{-k \mu t} t^{k-1} d(t) \\
& =\frac{\alpha k \mu \lambda^{n} k \mu^{(k-2)}}{n !(k-2) !} \int_{0}^{\infty} e^{-t(\lambda+k \mu)} t^{n+k-2} d(t) \\
& +\frac{\alpha k \mu \lambda^{n} k \mu^{(k-1)}}{n !(k-1) !} \int_{0}^{\infty} e^{-t(\lambda+k \mu)} t^{n+k-1} d(t) \\
& =\frac{\alpha \lambda^{n}(k \mu)^{k-1}}{n !(k-2) !} \int_{0}^{\infty} e^{-t(\lambda+k \mu)} t^{n+k-2} d(t)
\end{aligned}
$$

$$
\begin{aligned}
& +\frac{\alpha \lambda^{n}(k \mu)^{k}}{n !(k-1) !} \int_{0}^{\infty} e^{-t(\lambda+k \mu)} t^{n+k-1} d(t) \\
& =\frac{k \mu \lambda^{n} k \mu^{(k-1)}}{n !(k-1) !} \int_{0}^{\infty} e^{-t(\lambda+k \mu)} t^{n+k-1} d(t) \\
& \longrightarrow \text { Gama distribution } \Gamma(n+k) \\
& =\frac{\alpha \lambda^{n}(k \mu)^{k-1}}{n !(k-2) !}\left(\frac{1}{\lambda+k \mu}\right)^{n+k-1} \Gamma(n+k-1) \\
& +\frac{(1-\alpha) \lambda^{n}(k \mu)^{k}}{n !(k-1) !}\left(\frac{1}{\lambda+k \mu}\right)^{n+k} \Gamma(n+k) \\
& =\frac{(n+k-2) ! \alpha \lambda^{n}(k \mu)^{k-1}}{n !(k-2) !}\left(\frac{1}{\lambda+k \mu}\right)^{n+k-1} \\
& +\frac{(n+k-1) !(1-\alpha) \lambda^{n}(k \mu)^{k}}{n !(k-1) !}\left(\frac{1}{\lambda+k \mu}\right)^{n+k} \\
& =\left(\begin{array}{c}
n+k-2 \\
n
\end{array}\right)\left(\frac{1}{\lambda+k \mu}\right)^{n}\left(\frac{1}{\lambda+k \mu}\right)^{k-1} \alpha(k \mu)^{k-1} \lambda^{n} \\
& +\left(\begin{array}{c}
n+k-1 \\
n
\end{array}\right)\left(\frac{1}{\lambda+k \mu}\right)^{n}\left(\frac{1}{\lambda+k \mu}\right)^{k}(1-\alpha)(k \mu)^{k} \lambda^{n} \\
& =\alpha\left(\begin{array}{c}
n+k-2 \\
n
\end{array}\right)\left(\frac{\lambda}{\lambda+k \mu}\right)^{n}\left(\frac{k \mu}{\lambda+k \mu}\right)^{k-1} \\
& +(1-\alpha)\left(\begin{array}{c}
n+k-1 \\
n
\end{array}\right)\left(\frac{\lambda}{\lambda+k \mu}\right)^{n}\left(\frac{k \mu}{\lambda+k \mu}\right)^{k} .
\end{aligned}
$$

If the numerator and denominator are divided by $\mu$,

$$
\begin{aligned}
k_{n}= & \alpha\left(\begin{array}{c}
n+k-2 \\
n
\end{array}\right)\left(\frac{\lambda / \mu}{(\lambda+k \mu) / \mu}\right)^{n}\left(\frac{(k \mu) / \mu}{(\lambda+k \mu) / \mu}\right)^{k-1} \\
& +(1-\alpha)\left(\begin{array}{c}
n+k-1 \\
n
\end{array}\right)\left(\frac{\lambda / \mu}{(\lambda+k \mu) / \mu}\right)^{n}\left(\frac{(k \mu) / \mu}{(\lambda+k \mu) / \mu}\right)^{k} \\
= & \alpha\left(\begin{array}{c}
n+k-2 \\
n
\end{array}\right)\left(\frac{\rho}{\rho+k}\right)^{n}\left(\frac{k}{\rho+k}\right)^{k-1} \\
& +(1-\alpha)\left(\begin{array}{c}
n+k-1 \\
n
\end{array}\right)\left(\frac{\rho}{\rho+k}\right)^{n}\left(\frac{k}{\rho+k}\right)^{k}
\end{aligned}
$$

and cobrackets are taken as follows:

$$
\begin{aligned}
k_{n}= & \left.\frac{\rho}{\rho+k}\right)^{n}\left(\frac{k}{\rho+k}\right)^{k-1} \\
& \times\left(\alpha \frac{(n+k-2) !}{n !(k-2) !}\right. \\
& \left.\quad+(1-\alpha) \frac{(n+k-1)(n+k-2) !}{n !(k-1)(k-2) !}\left(\frac{k}{\rho+k}\right)\right)
\end{aligned}
$$




$$
\begin{aligned}
= & \left(\frac{\rho}{\rho+k}\right)^{n}\left(\frac{k}{\rho+k}\right)^{k-1} \frac{(n+k-2) !}{n !(k-2) !} \\
& \times\left(\alpha+(1-\alpha) \frac{(n+k-1)}{(k-1)}\left(\frac{k}{\rho+k}\right)\right) .
\end{aligned}
$$

$$
\begin{aligned}
& \times\left(\left(\frac{\rho_{0}}{\rho_{0}+k}\right)^{n}\left(\frac{k}{\rho_{0}+k}\right)^{k-1}\right. \\
& \left.\left.\quad \times\left(\alpha+(1-\alpha) \frac{(n+k-1)}{(k-1)}\left(\frac{k}{\rho_{0}+k}\right)\right)\right)^{-1}\right),
\end{aligned}
$$

Here

$$
\begin{gathered}
P_{i j}(\rho)=k_{j-i+1}, \quad i=1,2, \ldots, N, \\
j=0,1, \ldots, N-1, \quad j \geq i-1, \\
P_{0 j}(\rho)=k_{j}, \quad j=0,1, \ldots, N-1, \\
P_{0 N}(\rho)=1-\sum_{0}^{N-1} k_{n}, \\
P_{i N}(\rho)=1-\sum_{0}^{N-i} k_{n}, \quad i=1,2, \ldots, N, \\
=1-\sum_{r=0}^{N-i}\left(\frac{\rho}{\rho+k}\right)^{r}\left(\frac{k}{\rho+k}\right)^{k-1}\left(\begin{array}{c}
n+k-2 \\
n
\end{array}\right) \\
\times\left(\alpha+(1-\alpha) \frac{(r+k-1)}{(k-1)}\left(\frac{k}{\rho+k}\right)\right) .
\end{gathered}
$$

With these values for $P_{i j}(\rho)$, the logarithm of the likelihood ratio will be

$$
\begin{aligned}
\ln L & =\sum_{i, j} n_{i j} \ln \frac{P_{i j}\left(\rho_{1}\right)}{P_{i j}\left(\rho_{0}\right)} \\
& =a n+\sum_{i, j} n_{i j} c_{i j},
\end{aligned}
$$

$\ln L$

$$
\begin{aligned}
=\sum_{i, j} n_{i j} \ln ( & \left(\left(\frac{\rho_{1}}{\rho_{1}+k}\right)^{n}\left(\frac{k}{\rho_{1}+k}\right)^{k-1} \frac{(n+k-2) !}{n !(k-2) !}\right. \\
& \left.\times\left(\alpha+(1-\alpha) \frac{(n+k-1)}{(k-1)}\left(\frac{k}{\rho_{1}+k}\right)\right)\right) \\
& \times\left(\left(\frac{\rho_{0}}{\rho_{0}+k}\right)^{n}\left(\frac{k}{\rho_{0}+k}\right)^{k-1} \frac{(n+k-2) !}{n !(k-2) !}\right. \\
& \left.\left.\times\left(\alpha+(1-\alpha) \frac{(n+k-1)}{(k-1)}\left(\frac{k}{\rho_{0}+k}\right)\right)\right)^{-1}\right),
\end{aligned}
$$

$$
a=(k-1) \ln \left(\frac{\rho_{0}+k}{\rho_{1}+k}\right)
$$

$$
\begin{aligned}
& c_{i j}+b_{i j} \\
&= \ln \left(\frac{\rho_{1}}{\rho_{0}} \cdot \frac{\rho_{0}+k}{\rho_{1}+k}\right)^{j-i+1} \\
&+\ln \left(\frac{\alpha+(1-\alpha)((j-i+k) /(k-1))\left(k /\left(\rho_{1}+k\right)\right)}{\alpha+(1-\alpha)((j-i+k) /(k-1))\left(k /\left(\rho_{0}+k\right)\right)}\right), \\
& \quad i=1,2, \ldots, N, j=0,1, \ldots, N-1, j \geq i-1,
\end{aligned}
$$

Here

$$
\begin{aligned}
& c_{0 j}+b_{0 j} \\
&= \ln \left(\frac{\rho_{1}}{\rho_{0}} \cdot \frac{\rho_{0}+k}{\rho_{1}+k}\right)^{j} \\
&+\ln \left(\frac{\alpha+(1-\alpha)((j+k-1) /(k-1))\left(k /\left(\rho_{1}+k\right)\right)}{\alpha+(1-\alpha)((j+k-1) /(k-1))\left(k /\left(\rho_{0}+k\right)\right)}\right), \\
& j=0,1, \ldots, N-1,
\end{aligned}
$$

$c_{i N}$

$$
=\ln \left\{\left(1-\sum_{r=0}^{N-i}\left(\frac{\rho_{1}}{\rho_{1}+k}\right)^{r}\left(\frac{k}{\rho_{1}+k}\right)^{k-1}\left(\begin{array}{c}
r+k-2 \\
r
\end{array}\right)\right.\right.
$$

$\ln L$

$$
\begin{aligned}
=\sum_{i, j} n_{i j} \ln ( & \left(\frac{\rho_{1}}{\rho_{1}+k}\right)^{n}\left(\frac{k}{\rho_{1}+k}\right)^{k-1} \\
& \left.\times\left(\alpha+(1-\alpha) \frac{(n+k-1)}{(k-1)}\left(\frac{k}{\rho_{1}+k}\right)\right)\right)
\end{aligned}
$$

$$
\begin{gathered}
\left.\times\left(\alpha+(1-\alpha) \frac{(r+k-1)}{(k-1)}\left(\frac{k}{\rho_{1}+k}\right)\right)\right) \\
\times\left(1-\sum_{r=0}^{N-i}\left(\frac{\rho_{0}}{\rho_{0}+k}\right)^{r}\left(\frac{k}{\rho_{0}+k}\right)^{k-1}\left(\begin{array}{c}
r+k-2 \\
r
\end{array}\right)\right.
\end{gathered}
$$




$$
\begin{gathered}
\left.\left.\times\left(\alpha+(1-\alpha) \frac{(r+k-1)}{(k-1)}\left(\frac{k}{\rho_{0}+k}\right)\right)\right)^{-1}\right\} \\
i=1,2, \ldots, N, \\
c_{0 N}=c_{1 N} .
\end{gathered}
$$

\section{Numerical Results}

Consider a $M / H_{2} / 1$ queue (with poisson arrivals, two-phasetype exponential service, fixed $N$ ).

Let $\alpha_{1}=0.4$ and $\alpha_{2}=0.6$ be the probability for the upper phase and the probability for the lower phase and $\alpha=0.005$ and $\beta=0.05$ the first and second types of errors, respectively. Let $N=4$ be the capacity of the queuing system.

The mean value of $\rho$ is calculated using the following formula:

$$
\rho=\frac{\lambda}{\left(\left(\alpha_{1} / \mu_{1}\right)+\left(\alpha_{2} / \mu_{2}\right)\right)} .
$$

Suppose that we wish to maintain $\rho$ at the level 0.5 and we wish to detect whether its value has increased. Then, the hypothesis test is $H_{0}: \rho_{0}=0.5$ against alternative $H_{1}: \rho_{1}=$ 0.8 .

Let $t_{0}=0, t_{1}, t_{2}, t_{3}, \ldots$ be a discrete set of the number of customers remaining at points of departure in the $M / \mathrm{H}_{2} / 1$ queue. The number of customers remaining at the 48-point departure is given as follows:

00032101033221001321101000000

00010000001211002100 .

State space is $E=\{0,1,2,3\}$. Table 1 shows $n_{i j}$ that the number of transitions $i \rightarrow j$, and Table 2 shows the value of $c_{i j}$ calculating for SPRT.

From $\ln B<\sum_{i, j} n_{i j} c_{i j}<\ln A$ decision region, $\sum_{i, j} n_{i j} c_{i j}$ is between $\ln A$ and $\ln B$ so that $-2.9907<-1.1062<5.2470$. Therefore, the system is observed again and the number of customers remaining at the 49 points of departure is given as follows:

00011000000011110000001011010021

00000010000000000210022 .

Table 3 shows $n_{i j}$ that the number of transitions $i \rightarrow j$.

From $\ln B<\sum_{i, j} n_{i j} c_{i j}<\ln A$, the decision region, $\sum_{i, j} n_{i j} c_{i j}$, is small $\ln B$ so that $-4.3517<-2.9907$. Therefore, the hypothesis $H_{0}$ is accepted.

For the computation of the OC and ASN of the SPRT,

\begin{tabular}{|c|c|c|c|c|c|}
\hline \multirow{2}{*}{$i$} & \multicolumn{4}{|c|}{$j$} & \\
\hline & 0 & 1 & 2 & 3 & \\
\hline 0 & 17 & 5 & 1 & 2 & $n_{0}=25$ \\
\hline 1 & 8 & 2 & 1 & 1 & $n_{1}=12$ \\
\hline 2 & 0 & 5 & 1 & 0 & $n_{2}=6$ \\
\hline 3 & 0 & 0 & 3 & 1 & $n_{3}=4$ \\
\hline & & & & & $n=47$ \\
\hline
\end{tabular}
the largest latent root $\lambda_{0}(t, \rho)$ of the $P(t)$ matrix has been computed by fixing the values for $t$ and $\rho$. Figure 3 shows
TABLE 1

TABLE 2: The value of $c_{i j}$ calculating for SPRT.

\begin{tabular}{ccccc}
\hline$i$ & \multicolumn{3}{c}{$j$} & 2 \\
\hline 0 & -0.15871 & 0.114953 & 0.348346 & 0.744993 \\
1 & -0.15871 & 0.114953 & 0.348346 & 0.744993 \\
2 & 0 & -0.15871 & 0.114953 & 0.523806 \\
3 & 0 & 0 & -0.15871 & 0.280086 \\
\hline
\end{tabular}

$\ln L=\sum_{i, j} n_{i j} c_{i j}=-1.1062$.

TABLE 3

\begin{tabular}{ccccccc}
\hline$i$ & & & $j$ & & \\
\hline 0 & 0 & 1 & 2 & 3 & \\
1 & 12 & 11 & 4 & 2 & $n_{0}=59$ \\
2 & 0 & 6 & 1 & 1 & $n_{1}=24$ \\
3 & 0 & 0 & 3 & 1 & $n_{2}=9$ \\
\hline & & & & & $n=4$ \\
\hline
\end{tabular}

$\ln L=\sum_{i, j} n_{i j} c_{i j}=-4.35$.

the graphs of $\lambda_{0}(t, \rho)$ which have been plotted against the values of $t$ for different values of $\rho$.

As can be seen from Figure 3, there exists one and only one real $t_{0} \neq 0$ such that $\lambda_{0}\left(t_{0}\right)=1$. The derivative of $\lambda(t, \rho)$ at $t=0$ has been computed. The OC and ASN functions are then evaluated using the expressions between (13) and (17). The results of the OC and ASN functions for testing $H_{0}: \rho_{0}=$ 0.5 against $H_{1}: \rho_{1}=0.8$ are given in Tables 4 and 5 for $\alpha=$ $0.005, \beta=0.05$ and ve $\alpha=0.05, \beta=0.1$, respectively.

Figures 4 and 5 give the OC curve and the ASN curve for two different error probabilities in the same figure.

In this example, we wish to maintain $\rho$ at the level 0.5 and we wish to detect whether its value has increased. Then, the hypothesis test is $H_{0}: \rho_{0}=0.5$ against alternative $H_{1}$ : $\rho_{1}=0.8$. We accepted $H_{0}$ hypothesis. There is no need for any change in the system. So traffic intensity $(\rho)$ is the desired level. If any change in $\rho$, the queueing system would need to be organized. Namely, if $\rho$ has shifted to $\rho_{1}$ from $\rho_{0}\left(\rho_{1}>\rho_{0}\right)$, the control action could be to increase the mean $\mu$ or add an extra server. If $\rho$ has shifted to $\rho_{1}$ from $\rho_{0}\left(\rho_{1}<\rho_{0}\right)$, the control action could be to decrease the mean $\mu$ or reduce the number of servers.

We can understand from Table 4 that the number of samples required for this hypothesis to be accepted is 82.1395 


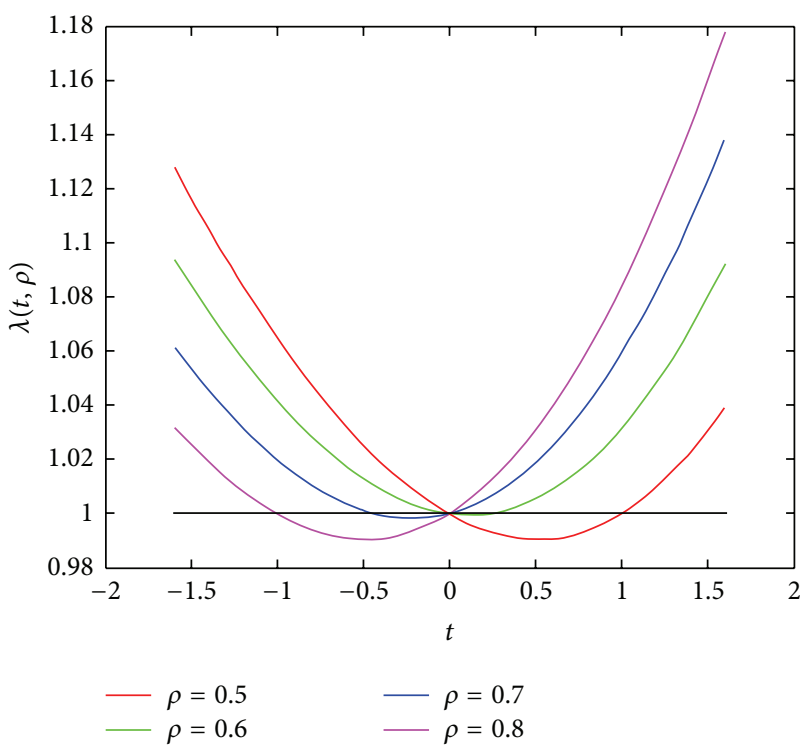

FIGURE 3: Graph of $\lambda(t, \rho)$ against $t$ for testing $H_{0}: \rho_{0}=0.5, H_{1}$ : $\rho_{1}=0.8$.

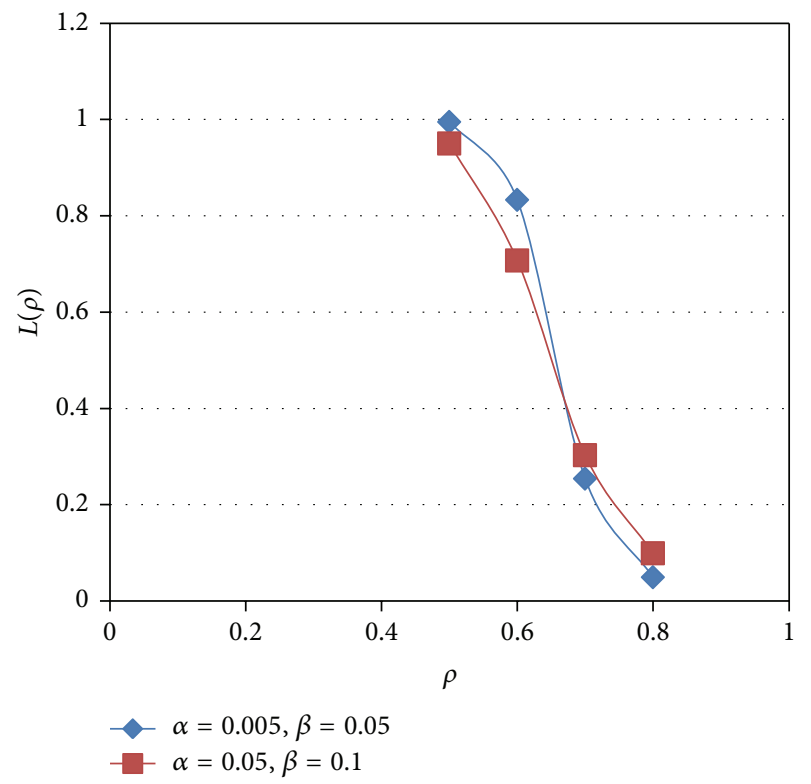

FIgure 4: The OC curve for testing $H_{0}: \rho_{0}=0.5$ against $H_{1}: \rho_{1}=$ 0.8 .

TABLE 4: The OC and ASN values for $\alpha=0.005$ and $\beta=0.05$.

\begin{tabular}{lcccc}
\hline$\rho$ & $t(\rho)$ & $\lambda_{0}^{\prime}(0, \rho)$ & $L(\rho)$ & $E(n, \rho)$ \\
\hline 0.5 & 1 & -0.0359 & 0.9951 & 82.1395 \\
0.6 & 0.2431 & -0.0087 & 0.8332 & 184.7906 \\
0.7 & -0.4308 & 0.0165 & 0.2543 & 191.3422 \\
0.8 & -1 & 0.0395 & 0.0496 & 122.4006 \\
\hline
\end{tabular}

and the number of samples required for it to be rejected is 122.4006 .

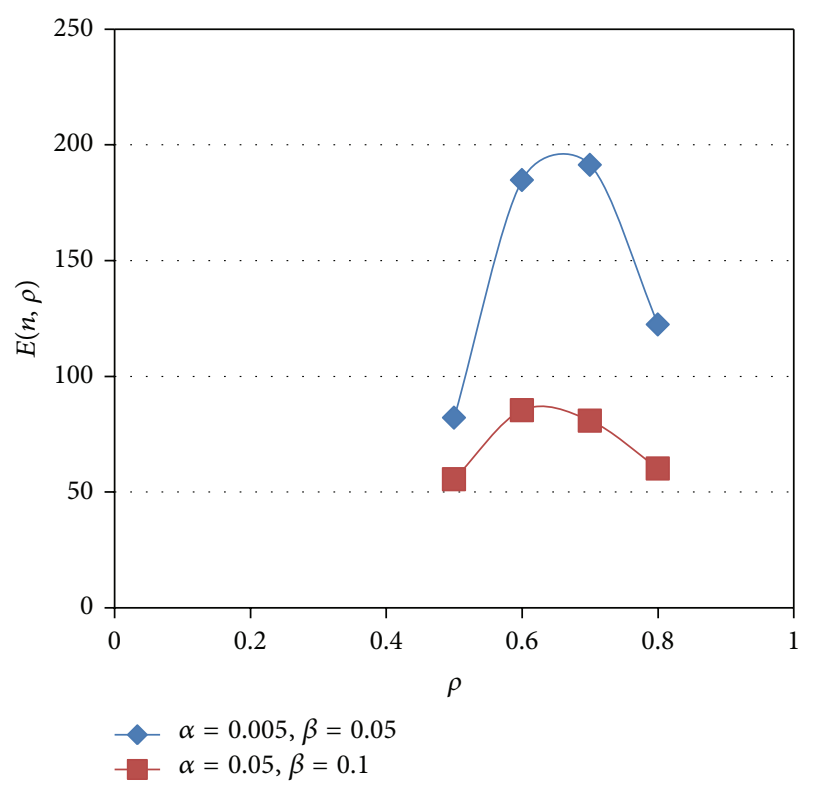

Figure 5: The ASN curve for testing $H_{0}: \rho_{0}=0.5$ against $H_{1}: \rho_{1}=$ 0.8 .

TABLE 5: The OC and ASN values for $\alpha=0.05$ and $\beta=0.1$.

\begin{tabular}{lcccc}
\hline$\rho$ & $t(\rho)$ & $\lambda_{0}^{\prime}(0, \rho)$ & $L(\rho)$ & $E(n, \rho)$ \\
\hline 0.5 & 1 & -0.0359 & 0.9503 & 55.5729 \\
0.6 & 0.2431 & -0.0087 & 0.7074 & 85.3826 \\
0.7 & -0.4308 & 0.0165 & 0.3031 & 80.8537 \\
0.8 & -1 & 0.0395 & 0.0994 & 60.1863 \\
\hline
\end{tabular}

As can be understood from the tables and Figures 4 and 5 , the smaller the error probability needed, the more the sampling number and power of a test are decreased.

\section{Conclusions}

The objective of the control technique is to detect changes in the traffic intensity $\rho$ as quickly as possible, then take appropriate corrective action, and determine how much of a sample size is needed in the applications. Thus, the sequential probability ratio test provides a saving of up to fifty percent in the sample size according to traditional methods. At the same time, the use of SPRT is easy for observing only the number of customers in the system at successive departure epochs $Q_{n}$, which are embedded Markov points.

In this paper, SPRT is theoretically investigated for two different phase-type queueing systems which consist of Hyperexponential and Mixed Erlang. Also the necessary coefficients have been obtained for SPRT.

In the application part, the queue lengths have been generated randomly for different arrival rates $(\lambda)$, different service rates $(\mu)$, and selected system capacities $(N)$ by using MATLAB 7.10.0 (R2010a) programming. Traffic intensities have also been calculated. With the obtained data and predetermined $\alpha$ and $\beta$, a simple hypothesis has been established. Accepting or rejecting the hypothesis has been examined by 
SPRT. Afterward, the largest latent root $\lambda(t, \rho)$ of the $P(t)$ matrix has been computed by fixing the values for $t$ and $\rho$. Their graphs have been drawn. It has been found that there exists one and only one real $t_{0} \neq 0$ such that $\lambda_{0}\left(t_{0}\right)=1$. The $\mathrm{OC}$ and ASN have been calculated with obtained values, and their graphs have been drawn by Microsoft Office Excel 2007 programming.

As a result, the length queue observed at the departure points can be maximum $N$, where $\rho>1$, and the length queue observed at the departure points can be minimum $N$, where $\rho<1$.

According to the given $\alpha$ and $\beta$, differences in sample size have been observed. It has been observed that when the error probability decreased the sample size grows and the power of test $(1-\beta)$ increases. Here, the value of $\mu$ needed to calculate the traffic intensity was taken from the mean $\mu$ (33). If you recall, the purpose of a control technique is to detect changes in the traffic intensity $\rho$. When $\rho$ has shifted to $\rho_{1}$ from the design level $\rho_{0}\left(\rho_{1}<\rho_{0}\right)$, an appropriate action is taken to bring $\rho_{1}$ back to the design level $\rho_{0}$. In the same way, when $\rho$ has shifted to $\rho_{1}$ from the design level $\rho_{0}$ $\left(\rho_{1}>\rho_{0}\right)$, an appropriate action is taken to bring $\rho_{1}$ back to the design level $\rho_{0}$. Consequently, the control action could be to increase mean $\mu$ or decrease mean $\mu$ for the phase-type queueing systems.

\section{References}

[1] R. M. Phatarfod, "Sequential analysis of dependent observations-I," Biometrika, vol. 52, pp. 157-165, 1965.

[2] U. N. Bhat and S. S. Rao, "A statistical technique for the control of traffic intensity in the queuing systems $M / G / 1$ and $G I / M / 1$," Operations Research, vol. 20, pp. 955-966, 1972.

[3] U. N. Bhat, "A statistical technique for the control of traffic intensity in Markovian queue," Annals of Operations Research, vol. 8, pp. 151-164, 1987.

[4] U. N. Bhat and S. S. Rao, "Statistical analysis of queueing systems," Queueing Systems, vol. 1, no. 3, pp. 217-247, 1987.

[5] K. Harishchandra and S. S. Rao, "Statistical inference about the traffic intensity parameter of $M / E_{k} / 1$ and $E_{k} / M / 1$ queues," Tech. Rep., Indian Institute of Management, Bangalore, India, 1984.

[6] S. S. Rao, U. N. Bhat, and K. Harishchandra, "Control of traffic intensity in a queue-a method based on SPRT," Opsearch, vol. 21, no. 2, pp. 63-80, 1984.

[7] S. Jain and J. G. C. Templeton, "Problem of statistical inference to control the traffic intensity," Sequential Analysis, vol. 8, no. 2, pp. 135-146, 1989.

[8] S. Jain, "An autoregressive process and its application to queueing mode," Metron-International Journal of Statistics, vol. 58, no. 1-2, pp. 131-138, 2000.

[9] A. Wald, Sequential Analysis, Dover, New York, NY, USA, 2004.

[10] S. S. Rao, U. N. Bhat, and K. Harishchanra, "OC and ASN of the SPRT for a finite markov chain and an application to the control of queues," Tech. Rep. 3, Indian Institute of Management, Bangalore, India, 1980.

[11] W. J. Stewart, Probability, Markov Chains, Queues, and Simulation, Princeton University Press, Princeton, NJ, USA, 2009. 


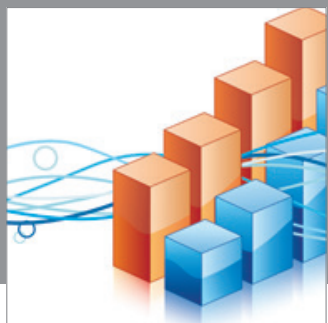

Advances in

Operations Research

mansans

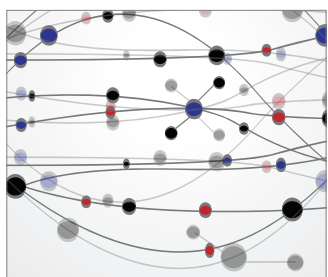

The Scientific World Journal
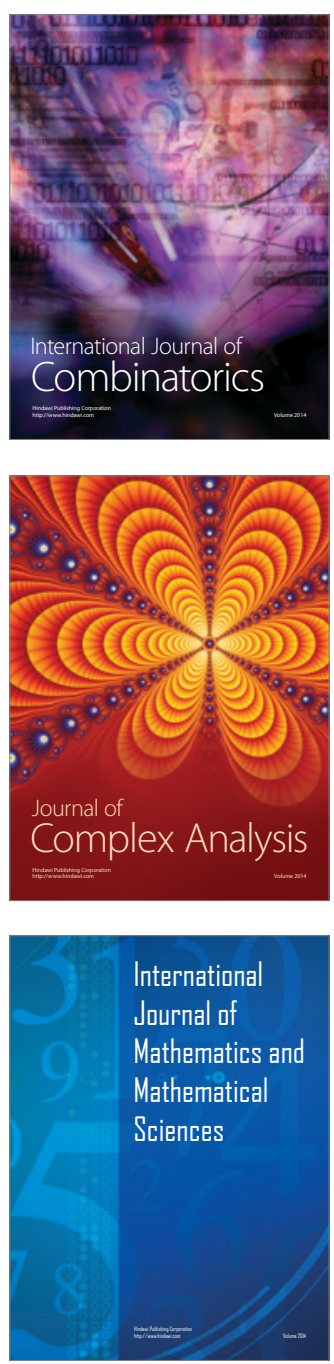
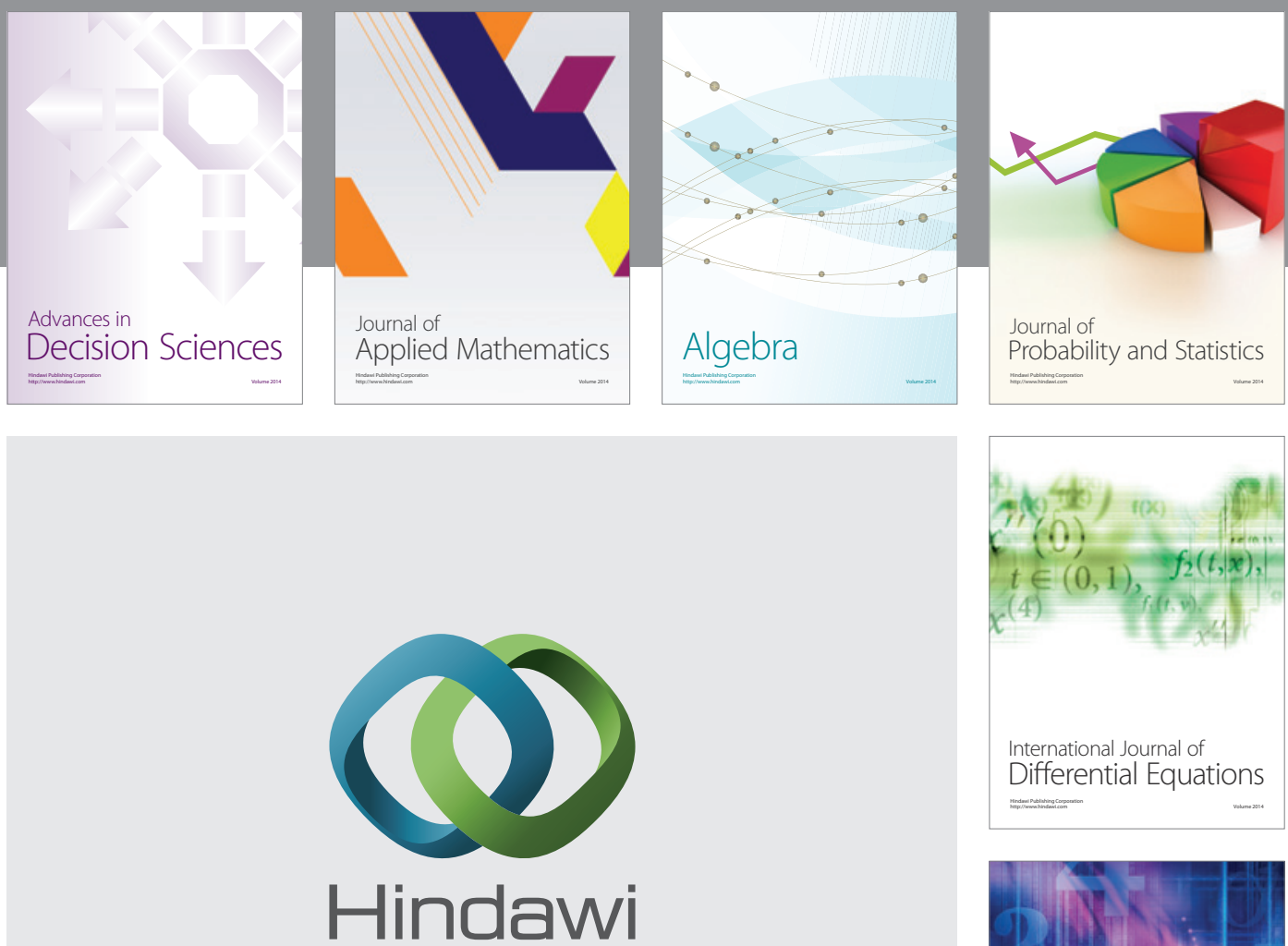

Submit your manuscripts at http://www.hindawi.com
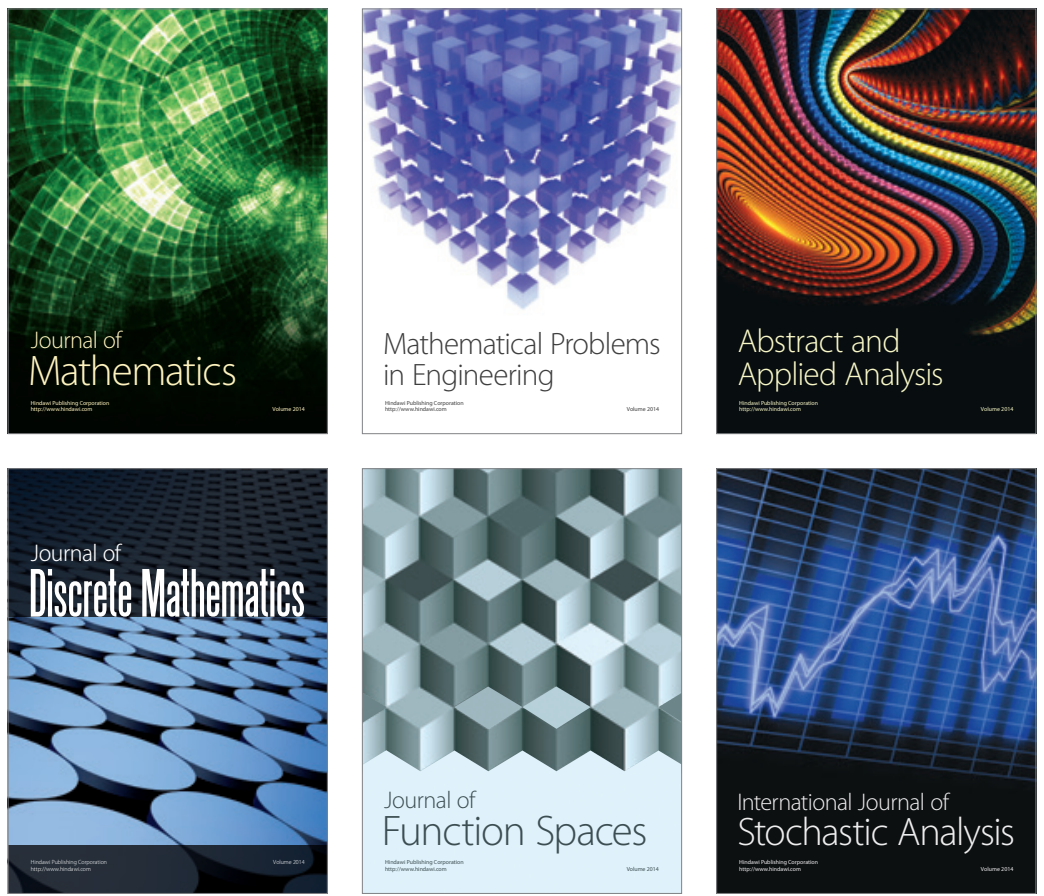

Journal of

Function Spaces

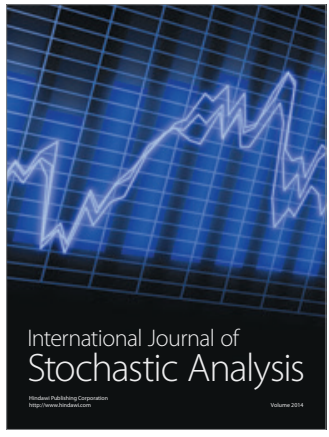

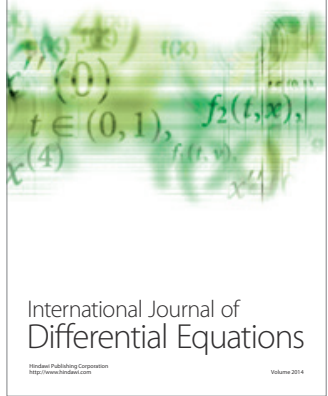
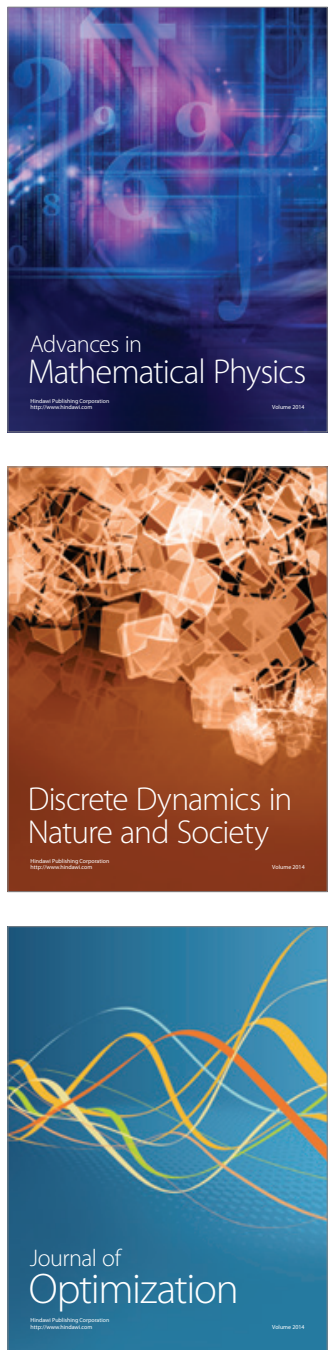